\title{
UTILIZAÇÃO CONJUNTA DE MODELAGEM ECONOMÉTRICA E OTIMIZAÇÃO EM DECISÕES DE CONCESSÃO DE CRÉDITO
}

\author{
Rodrigo Arnaldo Scarpel \\ Instituto Tecnológico de Aeronáutica - ITA \\ São José dos Campos - SP \\ Armando Zeferino Milioni * \\ Instituto Tecnológico de Aeronáutica - ITA \\ São José dos Campos - SP \\ E-mail: milioni@mec.ita.br \\ * Corresponding author/autor para quem as correspondências devem ser encaminhadas \\ Recebido em 08/2001, aceito em 02/2002 após 1 revisão
}

\begin{abstract}
Resumo
Utilizamos um modelo econométrico do tipo Logit como o desenvolvido por Scarpel \& Milioni (2001), concebido para atuar em previsão de insolvência de empresas, em conjunto com o modelo de programação linear inteira desenvolvido por Gehrlein \& Wagner (1997). O objetivo é o de dar suporte a decisões de concessão de crédito em função da estimativa da probabilidade de solvência de empresas, de forma a minimizar a soma dos custos de oportunidade e inadimplência. Mostramos que a utilização conjunta desses modelos possibilita a eliminação das limitações encontradas quando eles são utilizados isoladamente no auxílio às decisões de concessão de crédito.
\end{abstract}

Palavras-chave: crédito, Logit, programação em inteiros.

\begin{abstract}
We use a Logit Model such as the one developed by Scarpel \& Milioni (2001), designed to forecast corporations bankruptcy, together with an Integer Programming Model developed by Gehrlein \& Wagner (1997). We aim at supporting decisions of credit concession considering the corporation's solvency probability estimate and minimizing the sum of opportunity and failure to pay costs. As we show, the conjoint utilization of both models eliminates limitations found in each of them, when used in isolation.
\end{abstract}

Keywords: credit scoring, Logit, integer programming. 


\section{Introdução}

Uma grande variedade de métodos quantitativos vem sendo utilizada por instituições financeiras no auxílio à avaliação de risco de crédito com o objetivo de minimizar as perdas ocorridas devido a decisões equivocadas na concessão de crédito.

Rosenberg \& Gleit (1994) comentam que há muitas vantagens na utilização de métodos quantitativos em gerenciamento de crédito, destacando-se os benefícios resultantes da otimização no processo de tomada de decisão: fornece-se crédito (ou crédito adicional) aos melhores clientes (mais confiáveis), gerando aumento dos lucros e nega-se (ou diminui-se) o crédito aos piores clientes (menos confiáveis), resultando na diminuição das perdas. Além disso, políticas ótimas de cobrança minimizam os custos de administração e maximizam o montante recuperado do mal pagador.

A grande maioria das técnicas ou métodos quantitativos utiliza o sistema de pontuação, ou score. Por esse motivo, esses modelos são comumente chamados de modelos de credit scoring. Uma versão simples de um modelo de credit scoring é feita atribuindo-se pesos a algumas medidas, ou índices numéricos, buscando obter um somatório ponderado relacionado a cada solicitação de crédito. Um valor de corte é então especificado como instrumento de decisão, sendo que o crédito será fornecido para os clientes cujas solicitações apresentem um score superior ao valor de corte e negado caso contrário.

A análise discriminante é historicamente o método quantitativo mais utilizado na determinação dos pesos dos índices em modelos de credit scoring, sendo Altman (1968) o pioneiro na sua utilização. Ela é uma técnica estatística que permite estudar diferenças entre dois ou mais grupos, em função de um conjunto de informações conhecidas para todos os elementos dos grupos. Em gerenciamento de crédito é possível utilizar análise discriminante para avaliar se determinado cliente (pessoa física ou jurídica) é confiável ou não em termos de risco de crédito, sendo possível, também, estimar a magnitude desse risco, ou seja, se ele é pequeno ou grande. Todavia, segundo Rosenberg \& Gleit (1994), vários autores têm expressado ceticismo na utilização de análise discriminante em credit scoring. Segundo Eisensbeis (1977), o problema mais sério relacionado à utilização de análise discriminante em credit scoring relaciona-se à definição dos grupos. Isto porque os procedimentos utilizados em análise discriminante são apropriados quando se assume que os grupos são discretos e identificáveis, como, por exemplo, bons e maus clientes.

Modelos que utilizam outros métodos quantitativos são objetos de contínua investigação, sempre objetivando a melhoria das decisões de crédito. Dentre esses outros modelos estão os de programação linear inteira, como o desenvolvido por Gehrlein \& Wagner (1997) e os modelos econométricos, como os desenvolvidos por Scarpel (2000) e Scarpel \& Milioni (2001).

Neste trabalho, utilizamos um modelo econométrico do tipo Logit Binomial, como os desenvolvidos por Scarpel (2000) e Scarpel \& Milioni (2001), concebido para atuar em previsão de insolvência de empresas, em conjunto ao modelo de programação linear inteira desenvolvido por Gehrlein \& Wagner (1997). Nosso objetivo é o de conceber um modelo que forneça suporte a decisões de concessão de crédito em função da estimativa da probabilidade de solvência de empresas, de forma a minimizar a soma dos custos de oportunidade e inadimplência. Como veremos, a utilização conjunta desses modelos possibilita a eliminação de alguma das limitações encontradas quando eles são utilizados isoladamente. 
Na Seção 2 apresentamos o modelo de programação linear inteira de Gehrlein \& Wagner (1997). Na Seção 3 apresentamos o modelo Logit Binomial de Scarpel (2000) e Scarpel \& Milioni (2001). Essas duas seções terminam com um balanço resumido das principais faculdades e limitações de cada modelo. Na Seção 4 apresentamos nossa contribuição principal, que consiste numa modificação do modelo de Gehrlein e Wagner inspirada na formulação de Scarpel e Milioni. Nessa mesma seção, mostramos que a conjugação dos dois modelos auxilia na eliminação das limitações inerentes ao uso de cada um deles isoladamente, o que ilustramos numericamente na Seção 5. Finalmente, a Seção 6 é reservada às conclusões finais.

\section{Modelo de Programação Linear}

De acordo com Rosenberg \& Gleit (1994), modelos de programação linear inteira se constituem no segundo método quantitativo mais utilizado no auxílio às decisões de dar ou negar crédito. Esses modelos foram concebidos com uma preocupação inicial voltada para a capacidade de prognosticar e detectar potenciais não pagadores, ou seja, visando os casos onde se deseja evitar incorrer no custo relativo a emprestar dinheiro a clientes que não efetuarão o pagamento (custo de inadimplência). Posteriormente, incorporaram também o custo de oportunidade associado a negar empréstimo a clientes que efetuariam o pagamento.

Gehrlein \& Wagner (1997) desenvolveram uma formulação de programação linear inteira que minimiza a soma do custo de inadimplência com o custo de oportunidade. Essa formulação baseia-se num conjunto de $n$ observações históricas de antigos clientes, com um valor numérico para cada um dos $k$ atributos existentes na requisição de empréstimo feita inicialmente pelos clientes. Também é necessária a informação, para cada cliente, referente ao pagamento ou não do empréstimo contratado.

Para o cliente $i$, Aij refere-se ao valor numérico do atributo $j$, para $1 \leq j \leq k$. Seja $W j$ uma ponderação a ser designada ao atributo $j$ e $F(i), i=1,2, \ldots, n$, o credit score resultante, onde:

$$
F(i)=\sum_{j=1}^{k} W_{j} A_{i j}
$$

Um valor de corte $X c$ é utilizado para determinar se o cliente deve ou não receber o empréstimo. Assim, o empréstimo será fornecido quando $F(i)>X c$ e negado quando $F(i) \leq X c$.

Seja $d$ o conjunto de clientes que não pagou o empréstimo e $p$ o conjunto de clientes que pagou o empréstimo; seja $C d p$ o custo associado à classificação de um cliente que não pagaria o empréstimo como um cliente que pagaria e, simetricamente, $C p d$ o custo associado à classificação de um cliente que pagaria o empréstimo como um cliente que não pagaria. $\mathrm{O}$ modelo tem, então, a seguinte função objetivo:

$$
\operatorname{Min}\left[C d p \cdot \sum_{i \in d} I i+C p d \cdot \sum_{i \in p} I i\right]
$$

sujeito às restrições abaixo, onde $F(i)$ está definida na equação (1):

$$
\begin{aligned}
& F(i)+M . I i \geq X c+\varepsilon, \forall i \in p \\
& F(i)-M . I i \leq X c-\varepsilon, \forall i \in d
\end{aligned}
$$


Nas equações acima, $M$ é um número positivo e grande, $\varepsilon$ é um número positivo e pequeno e a variável binária $I i$ recebe valor 0 se a classificação do cliente é feita de forma correta e 1 se o cliente é classificado de forma incorreta.

Nesse tipo de modelo, a partir dos atributos Aij é possível estabelecer uma nota de corte $X c$ (que é variável de decisão) que permite a classificação dos clientes como explicado acima.

Uma notável faculdade do modelo acima - ver Gehrlein \& Wagner (1997) - é a de que ele permite o subsídio às decisões de fornecimento de crédito às empresas em função da política de juros estabelecida pelo concedente do crédito, uma vez que o modelo tem uma função objetivo que leva em consideração o custo de inadimplência e o custo de oportunidade dos empréstimos, minimizando a soma de ambos. Todavia, esse modelo tem como desvantagem, ou limitação, a adoção de uma escala própria de valores $(F i)$, conforme a equação (1), estabelecendo o valor de corte a partir dessa escala. Tal escala varia em função da ponderação atribuída às variáveis estabelecidas como discriminantes entre os clientes bons e maus pagadores (ou empresas solventes e insolventes). A adoção de uma escala própria de valores se constitui numa desvantagem, uma vez que não é possível, através da nota de corte Xc obtida pela aplicação do modelo de Gehrlein \& Wagner (1997), ter uma sensibilidade quanto à situação financeira da empresa, nem estimativas da probabilidade da empresa que está recorrendo ao crédito não efetuar o pagamento por motivos de insolvência. De fato, não é possível estabelecer uma interpretação econômica ou probabilística ao significado de $X c$.

\section{Modelo Logit}

De acordo com Pindyck \& Rubinfeld (1998), o modelo Logit Binomial (que doravante chamaremos apenas de Logit) é um modelo econométrico de seleção qualitativa, uma vez que gera respostas de procedimentos qualitativos do tipo presença ou ausência de um determinado atributo. Por exemplo, vota-se sim ou não em uma eleição, viaja-se de carro ou de avião, uma empresa irá ou não a falência, etc.

Trabalhos que utilizaram modelos Logit em auxílio às decisões de crédito não são muito comuns. Wiginton (1980) comparou o desempenho de um modelo Logit e um modelo que utilizou análise discriminante em credit scoring, concluindo que o modelo Logit teve um desempenho ligeiramente superior. Cheek (1994) calibrou um modelo Logit para estimar a probabilidade de instituições financeiras do Texas não receberem o pagamento pelos empréstimos concedidos em 1989 e Janot (2001) desenvolveu e examinou a eficácia de um modelo Logit e de um modelo de risco proporcional de Cox na previsão do fenômeno de insolvência bancária no Brasil durante o período 1995/1998.

O modelo Logit é baseado na função de probabilidade logística acumulada, a qual é especificada por:

$$
P i=F(Z i)=F\left(\alpha+\sum_{j} \beta_{j} \cdot X_{i j}\right)=\frac{1}{1+e^{-Z i}}=\frac{1}{1+e^{-\left(\alpha+\sum_{j} \beta_{j} \cdot X_{i j}\right)}}
$$

Nessa equação, $P i$ é a probabilidade de ocorrência de um evento dada a ocorrência de $X i j$ na observação $i$, para $1 \leq i \leq k$, onde $k$ é o número de observações existentes, $F($.) é a função distribuição acumulada, $\beta j$ é o coeficiente da variável independente $X i j$ e $Z i$ é um índice contínuo teórico determinado pelas variáveis explicativas $X i j$. 
A Figura 1 mostra o gráfico da função logística cumulada.

A partir de algumas transformações algébricas elementares sobre a equação (5) obtemos:

$$
L N\left(\frac{P i}{1-P i}\right)=Z i=\alpha+\sum_{j} \beta_{j} . X_{i j}
$$

A variável dependente da equação (6) é o logaritmo relacionado à probabilidade de ocorrência de determinado evento.

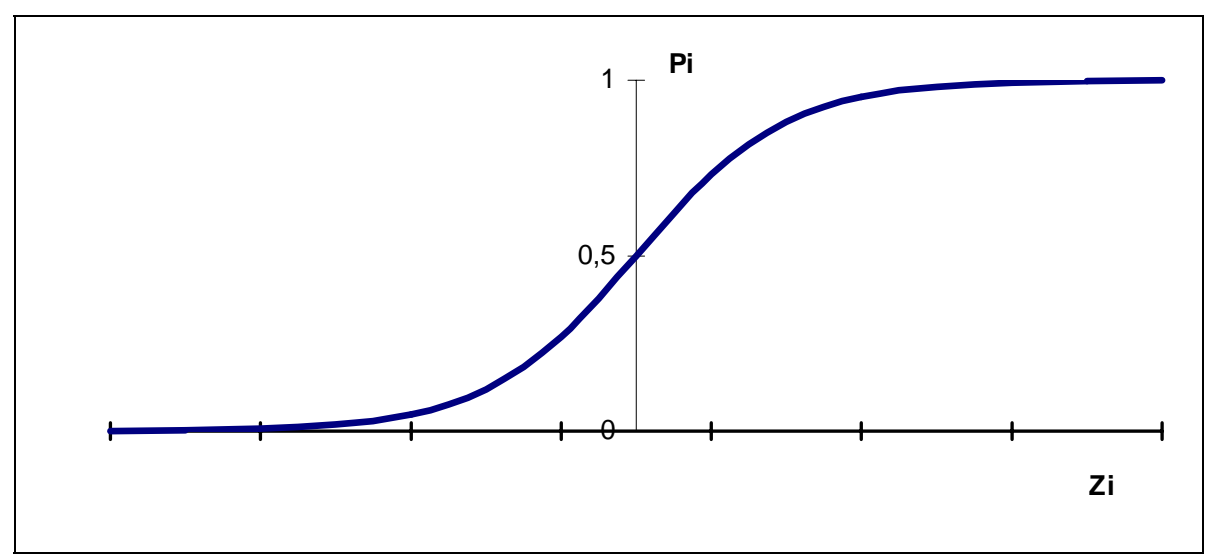

Figura 1 - Função logística acumulada

Em modelos com variáveis contínuas relacionadas a atributos, os quais servem como variáveis explicativas, é necessário estimar um modelo Logit no qual somente uma alternativa esteja associada a cada conjunto de valores assumido pelas variáveis independentes, ou seja, com observações individuais. Neste caso, a estimação dos parâmetros é feita pelo método da máxima verossimilhança - ver Scarpel (2000) - que é o mais recomendado quando dispomos de observações individuais da ocorrência ou não de determinado evento - ver, por exemplo, Pindyck \& Rubinfeld (1998).

Uma vantagem apresentada pelo modelo Logit, no caso do problema de classificação de empresas, é a de que ele permite fazer a classificação numa escala [0,1], estabelecendo de forma direta uma relação entre o valor obtido através do Logit e a probabilidade da empresa ser solvente/insolvente, e relacionando esse valor ao risco de não recebimento do empréstimo.

Em contrapartida, uma desvantagem ou limitação apresentada pelo modelo Logit quando comparado a outras ferramentas usadas em credit scoring é a de que ele não permite pautar as decisões de fornecimento de crédito pela política de juros adotada pelo fornecedor do crédito, ou seja, o modelo Logit não permite diferenciar instituições financeiras que cobram diferentes taxas de juros, como bancos de desenvolvimento (como por exemplo, o BNDES que em certos casos cobra uma taxa de juros de $12 \%$ ao ano) e financeiras (empresas de factoring), que chegam a cobrar uma taxa de juros de $12 \%$ ao mês (taxas em vigência no mercado brasileiro quando da elaboração do presente texto). 


\section{Utilização Conjunta dos Modelos Logit e de Programação Linear Inteira em Decisões de Concessão de Crédito}

Visando eliminar as limitações apresentadas pelos modelos Logit e de programação linear inteira quando utilizados de forma isolada, propomos o uso simultâneo das duas metodologias estabelecendo modificações no modelo de Gehrlein \& Wagner (1997).

A idéia central dessa modificação é a seguinte: ao invés de estabelecer uma função $F(i)$, calculada pela soma ponderada das variáveis discriminantes de empresas, utilizamos o modelo Logit. Começamos com a mesma função objetivo apresentada em (2), i.e.

$$
\operatorname{Min}\left[C d p \cdot \sum_{i \in d} I i+C p d \cdot \sum_{i \in p} I i\right]
$$

onde os conjuntos $d$ e $p$, os custos $C d p$ e $C p d$ e a variável binária $I i$ permanecem exatamente como definidos na Seção 2. Agora, entretanto, usando a equação (5), as restrições do modelo anteriormente apresentadas nas equações (3) e (4) passam a ser:

$$
\begin{aligned}
& P i+M . I i \geq V c+\varepsilon, \forall i \in p \\
& P i-M . I i \leq V c-\varepsilon, \forall i \in d
\end{aligned}
$$

Acima, $M$ e $\varepsilon$ permanecem como definidos anteriormente e $V c$ substitui $X c$ como variável de decisão do modelo, sendo o valor de corte utilizado para determinar se o cliente deve ou não receber o empréstimo. O empréstimo será fornecido quando $P i>V c$ e negado quando $P i \leq V c$.

Como $P i$ é uma probabilidade, $V c$ estará forçosamente contida no intervalo [0,1], ao contrário do que ocorria com $X c$, facultando sua interpretação como um valor limite de probabilidade de solvência a partir do qual o decisor aceita o risco de conceder o empréstimo. Por outro lado, pela manutenção da estrutura que contempla simultaneamente os dois custos simétricos $(C d p$ e $C p d)$, podemos construir cenários que incorporam a política de juros adotada pelo fornecedor do crédito.

\section{Ilustração de Uso do Modelo Modificado}

A seguir, ilustramos a utilização do modelo de programação linear inteira modificado, onde a função $F(i)$ foi substituída por $P i$, que é o valor da probabilidade de solvência calculado pelo modelo Logit.

Para tanto, faremos uso dos resultados obtidos por Scarpel \& Milioni (2001) que, utilizando o método da máxima verossimilhança, calibraram um modelo econométrico do tipo Logit para previsão de insolvência de empresas.

Scarpel \& Milioni (2001) objetivaram desenvolver uma ferramenta que servisse tanto para previsão de insolvência de empresas como para credit scoring, subsidiando decisões de dar ou negar crédito. Essas duas situações equivalem-se, de certa forma, uma vez que se tratando de fornecer ou negar crédito às empresas, é razoável supor que o crédito será negado quando existe o risco do não recebimento do empréstimo, ou seja, quando há evidências de que a empresa pode tornar-se inadimplente por motivos de insolvência. Nesse sentido, o modelo Logit desenvolvido presta-se tanto à previsão de insolvência quanto no auxilio às decisões de crédito. 
Quanto aos índices financeiros, buscando abranger as análises de liquidez, de atividade, de endividamento e de lucratividade, Scarpel \& Milioni (2001) selecionaram o índice mais representativo de cada uma dessas análises, de forma que o modelo construído fizesse uma análise completa a partir de índices normalmente utilizados e de amplo conhecimento. Assim, os índices financeiros selecionados foram o de liquidez corrente $(L C)$, o do giro do ativo total $(G A)$, o de endividamento geral $(E G)$ e o do retorno sobre o ativo total antes de pagamento de juros e imposto (ROA AJIR).

$\mathrm{O}$ índice de liquidez corrente $(L C)$ é calculado dividindo-se o ativo circulante (caixa, estoques e contas a receber) pelo passivo circulante (obrigações a vencer no próximo exercício). Portanto, é um índice que se relaciona de forma inversamente proporcional ao risco de curto prazo da empresa, pois indica quanto a empresa possui em dinheiro, em bens e direitos realizáveis no curto prazo, comparado com as dívidas a serem pagas no mesmo período.

O giro do ativo total $(G A)$ é um índice que relaciona à eficiência da empresa na utilização do seu ativo total na geração de receita, uma vez que é calculado dividindo-se a receita (ou vendas) total pelo ativo total. A interpretação do $G A$ é no sentido de quanto maior, melhor, uma vez que um baixo valor do giro de ativo indica que a empresa está gerando pouca receita a partir dos ativos que possui.

Já o índice de endividamento geral $(E G)$ é calculado pela razão entre o exigível total (= passivo circulante + exigível a longo prazo) e o ativo total, sendo do tipo quanto maior, pior, pois está relacionado, de forma diretamente proporcional, ao risco da empresa.

O ROA AJIR é, freqüentemente, chamado de retorno sobre o investimento da empresa. Ele mede a eficiência global da administração na geração de lucros a partir de seus ativos, antes de considerar os efeitos dos financiamentos. Esse índice é calculado dividindo-se o LAJIR (lucro antes do pagamento de juros e imposto de renda) pelo ativo total. Assim, quanto mais alto for esse índice, melhor.

A variável $L C$ não consta do modelo Logit obtido por Scarpel \& Milioni (2001) pois, como mostram os autores, houve multicolinearidade entre ela e o índice $E G$. Assim, houve a necessidade de retirar uma das duas variáveis e os autores optaram pela remoção de $L C$.

$\mathrm{Na}$ calibração foram utilizados dados de índices financeiros de 60 empresas brasileiras de capital aberto, das quais 28 eram insolventes e 32 eram solventes. A calibração feita foi validada com dados de 39 outras empresas cujos dados não foram utilizados na calibração. Nessa validação, o modelo previu corretamente a insolvência de todas as 7 empresas efetivamente insolventes e falhou prevendo a insolvência de 5 das 32 empresas que na verdade eram solventes. Dessa forma, sua eficiência global foi de $87 \%$, se considerarmos que o modelo previu a insolvência/solvência de forma correta de 34 das 39 empresas.

O resultado da calibração feita por Scarpel \& Milioni (2001) a partir do método da máxima verossimilhança foi o seguinte:

$$
P i=\frac{1}{1+E X P(-(0,190+3,312 * G A i-3,687 * E G i+10,997 * R O A A J I R i))}
$$

\subsection{Cenários}

Nesta ilustração contemplamos três cenários distintos: no primeiro, os custos de inadimplência e de oportunidade (respectivamente, Cin e Cop) são iguais; no segundo, o 
custo de inadimplência é maior do que o custo de oportunidade e no terceiro, o custo de inadimplência é menor do que o custo de oportunidade.

Na primeira situação, onde $\operatorname{Cin}=\operatorname{Cop}$, é de se esperar que o valor de corte obtido através da utilização do modelo de programação linear inteira modificado esteja próximo de 0,5 (ou $50 \%$ ), uma vez que a igualdade entre os custos de inadimplência e de oportunidade pode ser interpretada como se nenhuma diferenciação estivesse sendo feita em relação às taxas de juros cobradas pelos empréstimos, ou seja, não estaria sendo considerada a política de juros do fornecedor do crédito.

$\mathrm{Na}$ segunda situação, com Cin > Cop pretendemos exemplificar o caso de bancos de desenvolvimento que possuem políticas de juros baixos.

Finalmente, na terceira situação, com $C i n<C o p$ pretendemos exemplificar o caso de empresas financeiras que praticam taxas de juros elevadas.

Para efeito de ilustração, supomos um empréstimo de 100 unidades monetárias (u.m.) por um período de 24 meses e supomos ainda que em caso de inadimplência esta apenas ocorra depois do pagamento de $30 \%$ do principal emprestado (100 u.m.). Assim:

$$
\text { Cin }=70 \text { u.m. }
$$

O valor do pagamento mensal do empréstimos pode ser calculado a partir da fórmula:

$$
\text { Pagamento }=100 \cdot\left(\frac{t \cdot(1+t)^{24}}{(1+t)^{24}-1}\right)
$$

onde $t$ é a taxa de juros mensal cobrada pelo fornecedor do crédito e o fator entre parênteses é o fator de recuperação de capital de uma série uniforme de pagamentos - ver, por exemplo, Oliveira (1982). O custo de oportunidade pode ser obtido como segue:

$$
\text { Cop }=(24 * \text { Pagamento })-100
$$

O conjunto de dados de empresas utilizado na determinação dos valores de corte para o modelo de Gehrlein \& Wagner (1997) e para o modelo de programação linear inteira modificado estão apresentados nas Tabelas A1 e A2, no apêndice. Os mesmos também podem ser encontrados de maneira mais detalhada em Scarpel (2000). A equação Logit utilizada é aquela obtida por Milioni \& Scarpel (2001) e apresentada em (10).

\subsubsection{Custo de Oportunidade Igual ao Custo de Inadimplência}

Aplicando-se o modelo de programação linear inteira modificado obtivemos um valor de corte $(V c)$ de 0,435 , de tal forma que seria aprovado o crédito das empresas que tivessem probabilidade de ser solvente $(P i)$, resultante da aplicação do modelo Logit, superior a $43,5 \%$, negando-se o crédito às empresas com um valor de $P i$ inferior a 0,435 , ou seja, às empresas com uma probabilidade maior do que $56,5 \%$ de se tornarem insolventes. $\mathrm{O}$ valor obtido, que é naturalmente decorrente da massa de dados utilizada, pode ser considerado nas proximidades dos esperados 0,5 .

Aplicando-se o modelo de Gehrlein \& Wagner (1997), obtivemos um valor de corte (Xc) de 1,008 . 


\subsubsection{Custo de Inadimplência Maior que o Custo de Oportunidade}

Consideramos o caso em que a taxa de juros fosse de $12 \%$ ao ano, ou $0,949 \%$ ao mês. Nesse caso, a partir da equação (12) obtivemos para o pagamento mensal o valor de 4,68 u.m. Usando (13), temos o seguinte valor para o custo de oportunidade:

$$
\text { Cop }=(24 * 4,68)-100=12,27 \text { u.m. }
$$

Aplicando-se o modelo de programação linear inteira modificado obtivemos um valor de corte $(V c)$ de 0,796 , de forma que seria aprovado o crédito das empresas que tivessem uma probabilidade de ser solvente $(P i)$ superior a $79,6 \%$.

O valor obtido pode ser considerado coerente e consistente, uma vez que instituições financeiras que praticam baixas taxas de juros não podem arriscar-se fazendo empréstimos a quem tem uma probabilidade elevada de não honrar os pagamentos, de forma que os empréstimos seriam liberados apenas às empresas com baixa chance de tornarem-se insolventes.

Aplicando-se o modelo de Gehrlein \& Wagner (1997) obtivemos um valor de corte $(X c)$ de 2,495 .

\subsubsection{Custo de Oportunidade Maior que o Custo de Inadimplência}

Consideramos o caso em que a taxa de juros fosse de $12 \%$ ao mês. Nesse caso, a partir de (12) obtivemos para o pagamento mensal o valor de 12,85 u.m. A partir de (13), o custo de oportunidade fica:

$$
\text { Cop }=(24 * 12,85)-100=208,31 \text { u.m. }
$$

Aplicando-se o modelo de programação linear inteira modificado obtivemos um valor de corte $(V c)$ de 0,294 , de forma que seria aprovado o crédito das empresas que tivessem um valor de probabilidade de ser solvente $(P i)$ superior a $29,4 \%$.

Esse valor também pode ser considerado coerente e consistente, pois instituições financeiras que praticam altas taxas de juros podem arriscar-se mais, já que o custo de oportunidade de negar crédito a quem pagaria é superior ao custo de inadimplência.

Aplicando-se o modelo de programação linear inteira sem a modificação proposta obtivemos um valor de corte $(X c)$ de 0,418 .

A Tabela 1 resume as notas de corte obtidas através da aplicação dos modelos de programação linear inteira modificado $(V c)$ e não modificado $(X c)$ em cada um dos 3 cenários construídos.

Tabela 1 - Valor de corte obtido (modelos com e sem modificação)

\begin{tabular}{|c|c|c|c|}
\hline Cenário & Situação Simulada & $\boldsymbol{V} \boldsymbol{X}$ & $\boldsymbol{X}$ \\
\hline 1 & Desconsiderando a Taxa de Juros & 0,435 & 1,008 \\
\hline 2 & Taxa de Juros Baixa & 0,796 & 2,495 \\
\hline 3 & Taxa de Juros Elevada & 0,294 & 0,418 \\
\hline
\end{tabular}


Como vimos, não há uma interpretação econômica ou probabilística que possa ser associada aos valores apresentados na coluna $X c$ da Tabela 1. Em contrapartida, os valores apresentados na coluna $V c$ podem ser interpretados como a probabilidade de inadimplência a partir da qual a concessão de crédito é recomendável. Como era esperado, vemos que essa probabilidade caminha no sentido inverso ao da taxa de juros praticada e assume um valor intermediário, nas proximidades de $50 \%$, quando ela não é considerada.

\section{Conclusão}

Verificamos que a utilização conjunta dos modelos de programação linear inteira de Gehrlein \& Wagner (1997) e Logit de Scarpel \& Milioni (2001) (modelo de programação linear inteira modificado) possibilitou relacionar de forma direta a probabilidade da empresa tornarse insolvente, o risco do empréstimo e a taxa de juros praticada pela instituição fornecedora do crédito, sendo então estabelecido o valor mínimo da probabilidade de ser solvente necessário para a concessão do crédito.

Observamos ainda que quando variamos a taxa de juros cobrada pela instituição fornecedora de crédito os referidos valores mínimos mostraram-se coerentes pois, como é sabido, instituições financeiras que praticam baixas taxas de juros devem ser mais conservadoras na concessão de crédito, enquanto instituições financeiras que praticam altas taxas de juros podem, até certo ponto, correr maior risco, pois o custo de oportunidade de negar crédito a quem pagaria é superior ao custo de inadimplência.

\section{Referências Bibliográficas}

(1) Altman, E.I. (1968). Financial ratios, discriminant analysis, and the prediction of corporate bankruptcy. Journal of Finance, 23, 589-609.

(2) Cheek, G.E. (1999). Predicting savings and loan insolvency, <http://www.sbaer.eca.edu/docs/proceedings/94swi198.txt>

(3) Eisenbeis, R.A. (1977). Pitfalls in the application of discriminant analysis in business, finance, and economics. Journal of Finances, 32, 875-900.

(4) Gehrlein, W.V. \& Wagner, B.J. (1997). A two-stage least cost credit scoring model. Annals of Operations Research, 74, 159-171.

(5) Janot, M.M. (2001). Modelos de Previsão de Insolvência Bancária no Brasil. Banco Central do Brasil, Trabalhos para Discussão n.13.

(6) Oliveira, J.A.N. (1982). Engenharia Econômica: uma abordagem às decisões de investimento. McGraw-Hill, São Paulo, SP.

(7) Pindyck, R.S. \& Rubinfeld, D.L. (1998). Econometric models and economic forecasts. Irwin McGraw-Hill, Boston, Massachusetts.

(8) Rosenberg, E. \& Gleit, A. (1994). Quantitative methods in credit management: a survey. Operations Research, 42(4), 589-613.

(9) Scarpel, R.A. (2000). Modelos Matemáticos em Análise Financeira de Empresas, de Setores Industriais E de Crédito. Tese apresentada no Curso de Engenharia Aeronáutica e Mecânica do Instituto Tecnológico de Aeronáutica, São José dos Campos, SP. 
(10) Scarpel, R.A. \& Milioni, A.Z. (2001). Aplicação de modelagem econométrica à análise financeira de empresas. RAUSP - Revista de Administração da USP, 36, 80-88.

(11) Wiginton, J.C. (1980). A note on the comparison of Logit and discriminant models of consumer credit behavior. Journal on Finances and Quantitative Analysis, 15, 757-768.

\section{Apêndice}

Tabela A1 - Índices das empresas insolventes utilizados

\begin{tabular}{|c|c|c|c|}
\hline EMPRESA & GIRO ATIVO(GA) & ENDIV.GERAL(EG) & ROA AJIR \\
\hline 1 & 0,743 & 0,674 & 0,054 \\
\hline 2 & 0,267 & 0,637 & $-0,210$ \\
\hline 3 & 0,172 & 0,845 & 0,020 \\
\hline 4 & 1,661 & 0,601 & $-0,431$ \\
\hline 5 & 0,087 & 1,418 & $-0,347$ \\
\hline 6 & 0,046 & 0,279 & 0,018 \\
\hline 7 & 2,141 & 1,662 & $-0,608$ \\
\hline 8 & 0,508 & 0,976 & 0,018 \\
\hline 9 & 1,181 & 0,952 & $-0,028$ \\
\hline 10 & 1,302 & 0,723 & $-0,043$ \\
\hline 11 & 1,179 & 0,808 & $-0,224$ \\
\hline 12 & 0,875 & 3,541 & $-0,227$ \\
\hline 13 & 0,874 & 0,690 & 0,005 \\
\hline 14 & 0,950 & 0,972 & $-0,098$ \\
\hline 15 & 1,067 & 0,478 & $-0,097$ \\
\hline 16 & 0,497 & 0,801 & $-0,323$ \\
\hline 17 & 0,949 & 0,860 & $-0,362$ \\
\hline 18 & 0,263 & 1,000 & $-0,128$ \\
\hline 19 & 0,436 & 0,922 & $-0,087$ \\
\hline 20 & 0,480 & 0,902 & $-0,028$ \\
\hline 21 & 0,445 & 0,499 & $-0,025$ \\
\hline 22 & 0,067 & 0,679 & 0,056 \\
\hline 23 & 0,019 & 0,979 & $-0,490$ \\
\hline 24 & 0,000 & 0,889 & $-4,159$ \\
\hline 25 & 0,000 & 3,499 & $-0,704$ \\
\hline 26 & 0,210 & 0,792 & $-0,126$ \\
\hline 27 & 0,157 & 0,518 & $-0,235$ \\
\hline 28 & 0,804 & 0,792 & 0,074 \\
\hline
\end{tabular}


Tabela A2 - Índices das empresas solventes utilizados

\begin{tabular}{|c|c|c|c|}
\hline EMPRESA & GIRO ATIVO(GA) & ENDIV.GERAL(EG) & ROA AJIR \\
\hline 29 & 2,021 & 0,250 & 0,242 \\
\hline 30 & 0,630 & 0,641 & $-0,071$ \\
\hline 31 & 1,168 & 0,843 & 0,121 \\
\hline 32 & 0,957 & 0,559 & 0,048 \\
\hline 33 & 0,519 & 0,560 & 0,080 \\
\hline 34 & 0,196 & 0,151 & 0,298 \\
\hline 35 & 0,578 & 0,450 & $-0,024$ \\
\hline 36 & 0,302 & 0,106 & 0,121 \\
\hline 37 & 3,177 & 0,531 & 0,196 \\
\hline 38 & 1,515 & 0,600 & $-0,294$ \\
\hline 39 & 0,339 & 0,341 & 0,098 \\
\hline 40 & 0,790 & 0,248 & 0,082 \\
\hline 41 & 0,722 & 0,393 & 0,056 \\
\hline 42 & 0,629 & 0,680 & 0,157 \\
\hline 43 & 1,481 & 0,324 & 0,122 \\
\hline 44 & 1,551 & 0,918 & $-0,191$ \\
\hline 45 & 1,919 & 0,608 & $-0,023$ \\
\hline 46 & 2,320 & 0,368 & 0,121 \\
\hline 47 & 0,822 & 0,312 & 0,193 \\
\hline 48 & 1,267 & 0,337 & 0,224 \\
\hline 49 & 2,846 & 1,206 & 0,003 \\
\hline 50 & 1,452 & 0,520 & 0,106 \\
\hline 51 & 0,734 & 0,467 & $-0,021$ \\
\hline 52 & 0,499 & 0,198 & 0,047 \\
\hline 53 & 0,468 & 0,254 & 0,047 \\
\hline 54 & 0,796 & 0,129 & 0,111 \\
\hline 55 & 3,075 & 0,984 & 0,023 \\
\hline 56 & 0,691 & 1,038 & $-0,108$ \\
\hline 57 & 1,636 & 0,710 & 0,363 \\
\hline 58 & 1,869 & 1,322 & 0,110 \\
\hline 59 & 0,878 & 0,904 & $-0,002$ \\
\hline 60 & 0,978 & 1,210 & 0,147 \\
\hline
\end{tabular}

